\title{
RELATO DE óbitos maternos ocorridos de 2008 a 2013: relato de experiência no município de Porto Seguro, Bahia
}

\author{
Gestational risk classification based on maternal death profile 2008-2013: \\ an experience report from the municipality of Porto Seguro, Bahia, Brazil
}

Clasificación de riesgo gestacional basado en el perfil de óbitos maternos ocurridos de 2008 a 2013: relato de experiencia en el municipio de Porto Seguro, Bahia, Brasil

\author{
Márcia Maria dos Santos de Moraes ${ }^{1}$ - (D) orcid.org/0000-0001-5344-2337 \\ Marcia Alves Quaresma² - (D orcid.org/0000-0002-4969-773X \\ Urânia Souza de Jesus Oliveira² - (D) orcid.org/0000-0001-9672-1766 \\ Márcia Maria Pedreira da Silveira³ - (D) orcid.org/0000-0002-1880-0386 \\ 'Universidade Federal do Sul da Bahia, Centro de Formação em Saúde, Teixeira de Freitas, BA, Brasil \\ ${ }^{2}$ Secretaria Municipal de Saúde, Porto Seguro, BA, Brasil \\ ${ }^{3}$ Universidade Federal da Bahia, Maternidade Climério de Oliveira, Salvador, BA, Brasil
}

\section{Resumo}

Objetivo: retratar a criação de uma classificação de risco gestacional baseada no perfil dos óbitos maternos em município com alta ocorrência, e sua utilização no pré-natal. Métodos: o perfil foi elaborado utilizando-se fichas de óbitos maternos ocorridos em 2008-2013, considerando-se idade, escolaridade, raça/cor da pele, residência, doença preexistente e história reprodutiva. Resultados: o óbito materno foi mais frequente entre pardas/pretas, 30-39 anos de idade, baixa escolaridade, residência em distritos vulneráveis e doença cardíaca como principal patologia preexistente; o risco gestacional foi classificado a partir desse perfil, atribuindo-se pontos (1-3) para cada menor/maior frequência e definindo prioridades (P) assistenciais - PI=risco habitual (4-9pt: rotina para consultas/exames), PII=risco alto (10-16pt: reduzir tempo de espera para consultas/ exames em $50 \%$ ) e PIII=risco muito alto ( $\geq 17 \mathrm{pt}$ : acesso a consultas/exames em até 7 dias). Conclusão: a nova classificação sensibilizou os profissionais quanto aos determinantes envolvidos nesses óbitos e à necessidade de priorizar o acesso ao pré-natal conforme o risco.

Palavras-chave: Mortalidade Materna; Fatores de Risco; Assistência Pré-Natal; Acesso aos Serviços de Saúde; Estudos Transversais.

Endereço para correspondência:

Márcia Maria dos Santos de Moraes -Rua Isaac Azevedo, n 424, Condomínio Vista do Vale, São José, Teixeira de Freitas, Bahia, Brasil. CEP:45988-054

E-mail:dra.marciamaria@uol.com.br 


\section{Introdução}

A mortalidade materna (MM) continua a ser um dos maiores problemas de Saúde Pública. Ela foi definida pela Organização das Nações Unidas (ONU), em 2000, como indicador de uma das principais questões a serem enfrentadas no limite do novo milênio, com vistas à promoção do desenvolvimento social e humano e ao desaparecimento da extrema pobreza no mundo. Entre os Objetivos do Desenvolvimento do Milênio (ODM) propostos pela ONU, uma das metas a alcançar era a diminuição da MM em 75\% até o ano de 2015. 0 Brasil não alcançou essa meta, e os ODM foram substituídos pelos Objetivos do Desenvolvimento Sustentável (ODS). No caso do Brasil, a atual meta consiste em reduzir a razão de MM para 20/100 mil nascidos vivos (NV) até 2030. ${ }^{1}$

0 que chama a atenção quando se estuda a MM e se investigam suas causas é 0 alto potencial de evitabilidade desses óbitos quando ações são tomadas em tempo oportuno. Para conseguir melhora real da saúde materna, o foco não deve ser apenas no nexo causal, é necessário identificar e eliminar os obstáculos de acesso a serviços de saúde de qualidade, em todos os níveis do sistema sanitário. ${ }^{2}$ Alguns estudos evidenciam que 0 acesso aos serviços de saúde é essencial à redução das altas taxas de MM, e que os determinantes sociais, culturais e econômicos, ao lado das políticas de saúde, mantêm estreita relação com essas mortes. ${ }^{3,4}$

\section{0 conhecimento dos determinantes relacionados ao óbito materno em um município pode adicionar informações importantes à classificação de risco gestacional, de forma a se priorizarem ações assistenciais, em tempo oportuno, contribuindo com a prevenção de MM conforme a realidade local.}

McCarthy e Maine classificam os determinantes das mortes maternas em 'gerais', 'intermediários' e 'proximais'. ${ }^{5}$ Os determinantes gerais remetem a processos macrossociais, relativos às desigualdades socioeconômicas, discriminações étnico-raciais e ausência de políticas sociais dirigidas a segmentos menos privilegiados da população feminina. Entre os determinantes intermediários, destacam-se as restrições de acesso a bens sociais, enquanto os proximais estão representados pelas características sociodemográficas das mulheres e suas condições de saúde, além daqueles relacionados com o acesso aos cuidados, à facilidade de planejamento reprodutivo, e à assistência oportuna e de qualidade no pré-natal, no parto e no puerpério.

Nesse sentido, os profissionais atuantes na Atenção Primária à Saúde têm a oportunidade de efetivar diversas práticas capazes de contribuir para a prevenção da MM, operando nos determinantes proximais. Sabe-se que a assistência pré-natal não pode prevenir as principais complicações do parto, que são causas importantes de MM, porém algumas intervenções no pré-natal poderão favorecer o prognóstico materno e prevenir a MM. ${ }^{6}$

A atenção pré-natal pode controlar os fatores de risco que trazem complicações à gestação, além de permitir a detecção e o tratamento oportunos, contribuindo para um desfecho perinatal e materno favorável. ${ }^{7}$ É preciso lembrar, também, que aproximadamente $15 \%$ das gestações se caracterizam como de alto risco, e o pronto reconhecimento desses casos, associado à existência de retaguarda de serviços com maior complexidade e de um adequado acompanhamento, são decisivos para a manutenção da vida dessas mulheres. ${ }^{8}$

Os materiais técnicos relacionados à saúde da gestante, disponibilizados pelo Ministério da Saúde, ${ }^{9,10}$ orientam que a classificação do risco deve realizar-se na primeira consulta e nas subsequentes, favorecendo o acesso a um serviço com qualidade, por meio da identificação desses fatores o mais precocemente possível e de forma dinâmica, além de possibilitar o reconhecimento de situações que contribuem para mortes potencialmente evitáveis.

Apesar de esses instrumentos nortearem as equipes da Estratégia Saúde da Família (ESF) nas tomadas de decisões relacionadas à produção do cuidado e acompanhamento das gestantes no pré-natal de risco habitual, o conhecimento dos determinantes relacionados ao óbito materno em um município pode adicionar informações importantes à classificação de risco gestacional, de forma a se priorizarem ações assistenciais, em tempo oportuno, contribuindo com a prevenção de MM conforme a realidade local. Morse e colaboradores salientam que o estudo sobre os determinantes da MM ainda é falho no Brasil, em sua maioria de cunho descritivo, com identificação das causas básicas, avançando pouco na investigação dos fatores determinantes desses óbitos. ${ }^{11}$ 
No município de Porto Seguro, estado da Bahia, a manutenção de elevada razão de MM, desde 0 ano de 2009, motivou a vigilância epidemiológica do óbito (VEO) local a elaborar, em 2013, o Projeto Meta 35, com a missão de ampliar a integração com a Atenção Básica e promover uma aproximação com a rede hospitalar local, visando alcançar seu objetivo principal: intervir nas causas evitáveis de MM considerando os relatórios de investigação desses óbitos, tendo como meta a redução da razão de MM para um valor próximo de 35/100 mil NV até 2015, no cumprimento dos ODM.

Este relato teve como objetivo retratar a experiência com a criação de uma classificação de risco gestacional baseada no perfil dos óbitos maternos e sua utilização no pré-natal pelas equipes da Estratégia Saúde da Família em Porto Seguro, BA, Brasil.

\section{Métodos}

Para se traçar o perfil dos determinantes para MM, foram selecionadas fichas de investigação de óbitos maternos ocorridos - e investigados pela VEO municipal - no período de 2008 a 2013. Decidiu-se por partir de 2008, ano em que as investigações tiveram início. As fichas de investigação seguem os modelos preconizados pelo Ministério da Saúde, com informações hospitalares, ambulatoriais e domiciliares, assim como a ficha de conclusão da VEO. ${ }^{12}$

As características maternas gerais avaliadas foram: idade, escolaridade, ocupação, raça/cor da pele, distrito de residência, situação conjugal e renda mensal familiar. Para as condições de saúde e acessos, avaliou-se: presença de doença de base; número de gestações; história reprodutiva anterior; acesso habitual a consultas de pré-natal; acesso ao Pré-Natal de Alto Risco (PNAR) e a exames; idade gestacional na $1^{\mathrm{a}}$ consulta; tipo de parto; causa da morte; e condições de nascimento do recém-nascido/feto. Os dados foram tabulados em planilha da plataforma Excel e descritos por meio de frequências. As fichas de investigação de óbitos são instrumentos de trabalho diário dos técnicos da VEO, que emitem relatórios anuais com informações agregadas, sem possibilidade de identificação individual. Esses relatórios são divulgados para a vigilância epidemiológica, a Atenção Básica municipal, coordenadores, gestores e comitês de óbito, e são de acesso ao público. Por estes motivos, o estudo não foi submetido a um Comitê de Ética em Pesquisa. ${ }^{13}$

\section{Resultados}

No período estudado, ocorreram 23 óbitos maternos, dos quais $73,9 \%$ por causas obstétricas diretas/ indiretas, e os demais $(26,1 \%)$ por morte materna tardia. A seleção das variáveis, que formaram a classificação de risco, teve como base os determinantes mais destacados, além daqueles que caracterizaram vulnerabilidades do município, como, por exemplo, região ou distrito de saúde (Tabela 1 ).

Sobre o total de óbitos maternos, a Tabela 1 mostra maior frequência para a faixa etária de 30 a 39 anos $(52,2 \%)$, mulheres com menos de oito anos de estudo $(47,8 \%)$ e pretas/pardas $(73,9 \%)$. Os distritos sanitários prevalentes foram aqueles correspondentes às áreas de maior vulnerabilidade social. Estes achados apresentam resultados semelhantes aos encontrados em outros estudos avaliativos dos determinantes envolvidos na MM. . $^{11,14-16}$

Observou-se que 56,3\% das mulheres tinham alguma patologia de base e a doença cardíaca despontou como a mais frequente. Das 23 mortes, em 13 havia história reprodutiva para alguma doença ou agravo, representando um número expressivo de mulheres que necessitaram de atenção mais individualizada (Tabela 2). Entretanto, mais da metade (56,5\%) fez apenas 4 a 6 consultas de pré-natal (Tabela 1).

As principais causas obstétricas diretas foram as hemorragias e as infecções puerperais; doenças do aparelho circulatório complicadas pela gravidez, parto e puerpério destacaram-se entre as causas indiretas dos óbitos maternos.

Com base nos resultados sobre perfil, seis variáveis se destacaram e foram selecionadas para a construção da classificação de risco gestacional do município: idade materna, escolaridade, raça/cor da pele, distrito sanitário, doença de base e história reprodutiva anterior.

As frequências obtidas, para cada categoria, receberam pontuação de $1 \mathrm{a} 3$, sendo 3 pontos para a faixa de maior frequência e 1 ponto para a menor (Tabela 3). Para a categoria 'anos de estudo', apesar de não haver registro na faixa 'não alfabetizada', esta recebeu a mesma pontuação que a faixa ' 1 a 3 anos', dada a relevância dessa condição como determinante para morte materna. A dificuldade das equipes em valorizar o histórico reprodutivo das gestantes fez com que essa categoria fosse considerada como de importância isolada, recebendo também a pontuação máxima (3 pontos) para qualquer resultado. 
Tabela 1 - Características das mortes maternas, segundo idade, anos de estudo, raça/cor da pele, distrito de saúde e número de consultas de pré-natal, ocorridas no município de Porto Seguro, Bahia, 2008-2013

\begin{tabular}{|c|c|}
\hline Características & $\mathbf{N}$ \\
\hline \multicolumn{2}{|c|}{ Idade materna (em anos) } \\
\hline 10 a 14 & - \\
\hline 15 a 19 & 5 \\
\hline 20 a 29 & 6 \\
\hline 30 a 39 & 12 \\
\hline 40 a 49 & - \\
\hline \multicolumn{2}{|l|}{ Anos de estudo } \\
\hline 1 a 3 & 2 \\
\hline 4 a 7 & 9 \\
\hline 8 a 11 & 10 \\
\hline 12 ou mais & - \\
\hline Não alfabetizada & - \\
\hline Anos ignorados & 2 \\
\hline \multicolumn{2}{|l|}{ Raça/cor da pele } \\
\hline Branca & 2 \\
\hline Indígena & 3 \\
\hline Parda & 12 \\
\hline Preta & 5 \\
\hline Raça/cor Ignorada & 1 \\
\hline \multicolumn{2}{|c|}{ Região de saúde (distritos sanitários) } \\
\hline Centro & 3 \\
\hline Frei Calixto & 8 \\
\hline Litoral Sul & 8 \\
\hline Zona rural & 1 \\
\hline Aldeias indígenas & 3 \\
\hline \multicolumn{2}{|c|}{ Consultas de pré-natal } \\
\hline 1 a 3 & 5 \\
\hline 4 a 6 & 13 \\
\hline 7 ou mais & 4 \\
\hline Não fez pré-natal & 1 \\
\hline
\end{tabular}

Nota: ( - ) Quando se trata de dado numérico igual a zero, não resultante de arredondamento.

A pontuação final era representada pela soma dos pontos das seis categorias analisadas. Dessa forma, o risco gestacional foi classificado em habitual ( 4 a 9 pontos), alto (10 a 16 pontos) ou muito alto (17 pontos ou mais) (Figura 1).

A partir dessa nova classificação, a prioridade na assistência pré-natal foi determinada conforme a graduação do risco gestacional apontado. Para o Risco Habitual foi estabelecida a Prioridade I, de que a gestante deveria entrar na rotina da equipe para consultas e exames. Na Prioridade II, o tempo de espera para consulta e exames do pré-natal deveria se reduzir em $50 \%$, e para a Prioridade III, 0 acesso às consultas e exames não deveria ultrapassar sete dias (Figura 1).

Previamente à distribuição do instrumento, foram realizadas reuniões com a coordenação da Atenção Básica e da regulação da Assistência, para pactuação dos prazos das consultas com especialistas focais e exames, conforme as prioridades estabelecidas. 
Tabela 2 - Características das mortes maternas segundo doença de base e história reprodutiva, ocorridas no município de Porto Seguro, Bahia, 2008-2013

\begin{tabular}{ll}
\hline Características & N \\
\hline Doença de base/agravo & 5 \\
\hline Cardíaca & 2 \\
Dependência química/álcool & 2 \\
Neurológica & 1 \\
Digestiva & 1 \\
Endócrina & 1 \\
Hematológica & 1 \\
Respiratória & 6 \\
Sem doença prévia & 4 \\
Ignorada & 23 \\
\hline Total & \\
\hline História reprodutiva & 8 \\
\hline Abortamento & 2 \\
Morte perinatal & 1 \\
Síndrome hemorrágica & 1 \\
Pré-eclâmpsia & 13 \\
\hline Depressão & 1 \\
\hline Total & 13 \\
\hline Nota:A diferença entre a soma das parcelas e o total em percentual decorre do critério de arredondamento. & 1 \\
\hline
\end{tabular}

As equipes foram sensibilizadas para a situação de morte materna no município, com apresentação do resultado do estudo sobre o perfil. Elas também foram esclarecidas quanto à forma como a classificação de risco gestacional foi construída e foram treinadas no uso correto do instrumento. Este foi distribuído impresso em cores, em papel no tamanho A4, plastificado, apresentando um texto instrutivo com orientações sobre a forma de pontuar as categorias listadas, estabelecer o grau de risco e a prioridade na assistência.

Durante a consulta de pré-natal na Atenção Básica, a gestante era avaliada para 0 risco gestacional com base no instrumento, recebendo uma pontuação final, classificação e definição de prioridade assistencial.

Para evitar que a gestante fosse identificada pelo termo 'risco', tomou-se o cuidado de sinalizar o cartão de pré-natal e as requisições de consultas e exames com a cor azul, amarela ou rosa, significando risco habitual, alto ou muito alto, respectivamente (Figura 1). A rede de atenção à gestante também recebeu orientações quanto ao significado das cores.
Para os casos encaminhados a outros serviços, houve preocupação com a integralidade da assistência, mantendo-se o acompanhamento das gestantes mesmo com o efetivo acolhimento no serviço referenciado.

\section{Discussão}

A partir das primeiras reuniões com as equipes, foi possível observar que os instrumentos disponibilizados pelo Ministério da Saúde eram pouco utilizados na rotina de atendimento ao pré-natal. Com a apresentação da nova classificação, houve maior sensibilização e cuidado dos profissionais com o uso das ferramentas disponíveis para auxiliar na melhoria do cuidado às gestantes.

As equipes demonstraram consciência da importância do reconhecimento dos determinantes envolvidos na morte materna. A prática de registrar dados sobre escolaridade e raça/cor da pele não fazia parte da rotina das equipes. Elaborada e publicada pelo Ministério da Saúde, a Caderneta da gestante, em sua versão 2016, ${ }^{17}$ não traz o item 'escolaridade' na identificação da gestante. A necessidade da busca 
Tabela 3 - Distribuição das categorias e respectivas pontuações para cálculo do risco gestacional no município de Porto Seguro, Bahia

\begin{tabular}{|c|c|c|c|}
\hline Categorias & Pontuação & Categorias & Pontuação \\
\hline Idade materna (em anos) & & Doença de base/agravo & \\
\hline 10 a 14 & 1 & Cardíaca & 3 \\
\hline 15 a 19 & 2 & Dependência química/álcool & 2 \\
\hline 20 a 29 & 2 & Neurológica & 2 \\
\hline 30 a 39 & 3 & Digestiva & 2 \\
\hline 40 a 49 & 1 & Endócrina & 2 \\
\hline Anos de estudo & & Hematológica & 2 \\
\hline Não alfabetizada & 3 & Respiratória & 2 \\
\hline 1 a 3 & 3 & Outra & 2 \\
\hline 4 a 7 & 2 & Tabagismo & 1 \\
\hline 8 a 11 & 1 & & \\
\hline 12 ou mais & 1 & & \\
\hline Raça/cor da pele & & História reprodutiva & \\
\hline Branca & 1 & Abortamento & 3 \\
\hline Indígena & 2 & Morte perinatal & 3 \\
\hline Parda & 3 & Síndrome hemorrágica & 3 \\
\hline Preta & 3 & Pré-eclâmpsia & 3 \\
\hline $\begin{array}{l}\text { Região de saúde } \\
\text { (distritos sanitários) }\end{array}$ & & $\begin{array}{l}\text { Depressão } \\
\text { Outra }\end{array}$ & $\begin{array}{l}3 \\
3\end{array}$ \\
\hline Centro & 2 & & \\
\hline Frei Calixto & 3 & & \\
\hline Litoral Sul & 3 & & \\
\hline Zona rural & 1 & & \\
\hline Aldeias indígenas & 2 & & \\
\hline
\end{tabular}

por essa informação foi apontada pelos profissionais como fator indicativo da importância da completude dos dados nos prontuários.

A partir do relato das equipes, foi possível observar que a avaliação cardiológica era insuficiente. Encaminhamentos ao cardiologista ocorriam quando a gestante manifestasse conhecimento de ser portadora de alguma doença do sistema cardiovascular preexistente à gravidez, ou quando um quadro clínico de descompensação cardíaca surgisse. Com a nova classificação, a abordagem clínica das condições cardiológicas passou a ser mais detalhada, e o exame de eletrocardiograma, de mais fácil acesso às gestantes.

0 histórico reprodutivo da gestante não era valorizado, levando a uma deficiência na identificação das situações de risco presumido para complicações obstétricas. Com a nova classificação e sensibilização, os profissionais passaram a valorizar mais esse tópico na anamnese obstétrica, e a colher informações mais detalhadas dos antecedentes ginecológicos e obstétricos.

A priorização na assistência pré-natal, de acordo com o perfil da gestante e graduação do risco, produziu efeitos positivos nas equipes. Estas relataram encontrar (i) maior segurança na condução dos casos em tempo oportuno e (ii) garantia de acesso aos exames e consultas, superando as fragilidades dos encaminhamentos.

A manutenção de elevadas razões de MM no município, desde o ano de 2009, motivou o desenvolvimento desta experiência. 0 Projeto Meta 35 representava um chamamento aos profissionais e gestores envolvidos na atenção à gestante, diante das informações descritas nos relatórios das investigações de óbitos maternos que mostravam a necessidade de investimentos em ações com um olhar mais direcionado para a Atenção Primária. 


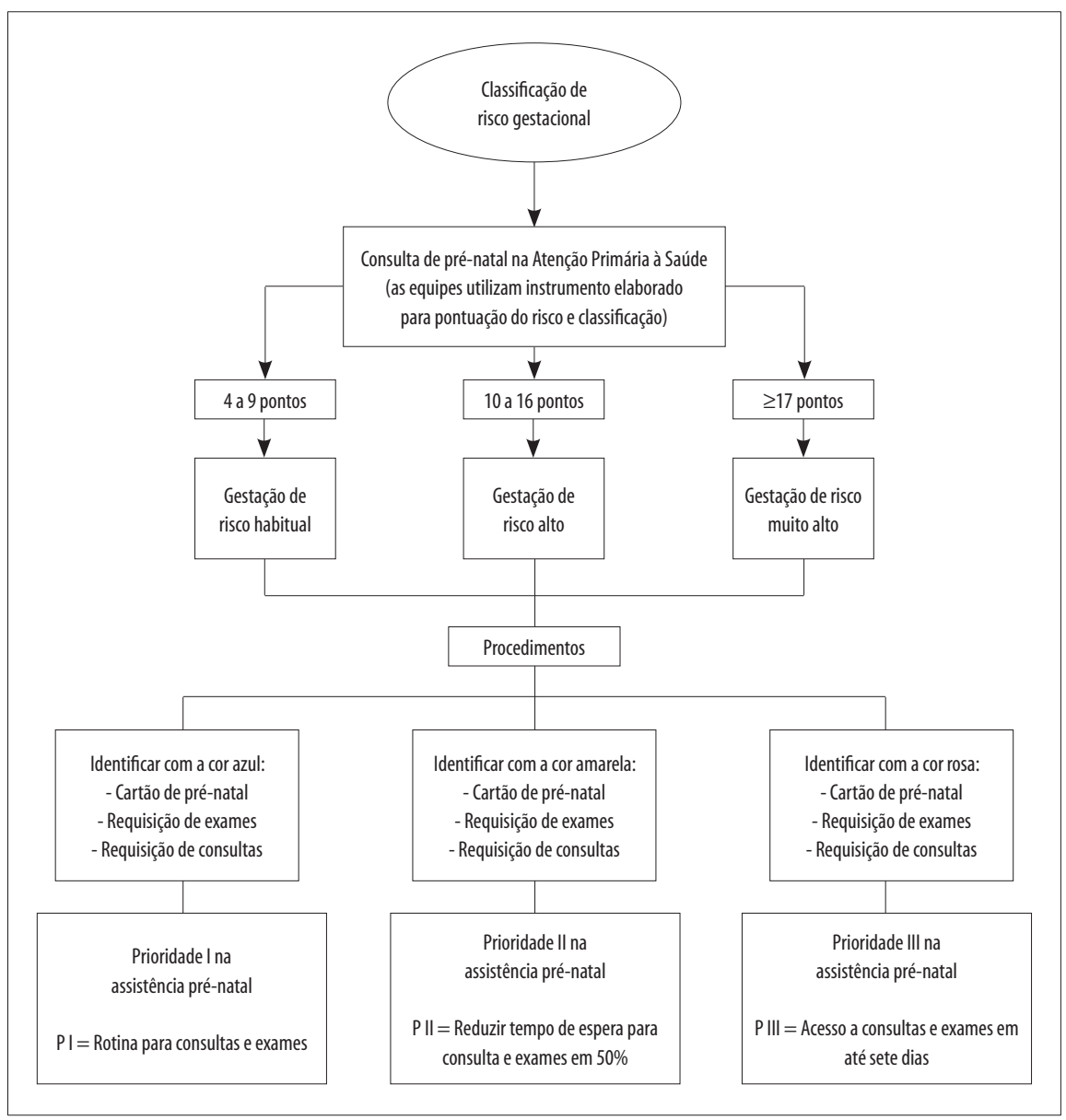

\section{Figura 1 - Fluxograma do atendimento pré-natal com classificação de risco gestacional}

Foi um projeto desafiador, face à delicada situação de mortes maternas em que o município se encontrava.

Desde 2007, a VEO atua de forma integrada com a Atenção Básica na prevenção da mortalidade materna e infantil, e considera essa parceria de fundamental importância para a consecução das ações de prevenção de agravos e promoção da saúde. A sensibilização das equipes, sua integração com a vigilância do óbito e o apoio da gestão foram fundamentais para o sucesso da experiência.

Traçar o perfil epidemiológico e expor/elucidar a situação da MM, destacando os determinantes específicos para o município, foi parte relevante do projeto. A complexidade dos determinantes envolvidos na morte materna representa um importante desafio para a gestão, pela necessidade de se articularem ações intersetoriais. Contudo, ao se considerar o elevado percentual de mortes por causas evitáveis, reconhece-se, mais do que a possibi- lidade, a necessidade de construir estratégias que tragam sinais de mudança para esse cenário de altos índices de mortes presumidas. A prevalência da baixa escolaridade, a raça/cor da pele parda ou preta e a moradia em áreas com maior vulnerabilidade social evidenciam a manutenção das iniquidades relacionadas à MM.

A maioria dos estudos sobre causas obstétricas de MM trazem as causas diretas (distúrbios hipertensivos, hemorragias e infecções puerperais) como as mais frequentes. ${ }^{11,15,18,19}$ Neste estudo, as causas indiretas corresponderam a uma proporção importante de óbitos, secundando as hemorragias. Contudo, independentemente da causa, os óbitos chamam a atenção para o potencial de evitabilidade. Martins e Silva, ao analisarem o perfil de mortes maternas, encontraram um percentual de $73 \%$ de óbitos evitáveis, ou provavelmente evitáveis, se intervenções fossem realizadas em tempo oportuno. ${ }^{19}$ 
A classificação de risco, construída com base no perfil de MM do município de Porto Seguro, e a definição de prioridades assistenciais, trouxeram oportunidades à gestão de reorganizar a utilização de recursos e assim melhorar os cuidados prestados à gestante.

De acordo com estudo recente, sobre internações por complicações obstétricas na gestação e desfechos maternos e perinatais no município de São Paulo, gestantes que apresentaram complicações obstétricas tiveram desfechos mais desfavoráveis da gestação, como internação pós-parto e MM, além de maior readmissão hospitalar dos recém-nascidos, concluindo que esses eventos devem ser considerados eventos sentinelas no monitoramento da assistência ao parto e ao recém-nascido na população atendida pelo Sistema Único de Saúde (SUS) ${ }^{20}$

Para a VEO, a experiência-objeto deste relato consagrou os processos de trabalho pautados na construção e compartilhamento de informações e na recomendação de procedimentos, contribuindo para que as práticas dos profissionais de saúde da Atenção Básica sejam articuladas com as ações de vigilância em

\section{Referências}

1. Organização das Nações Unidas. Transformando nosso mundo: a agenda 2030 para o desenvolvimento sustentável [Internet]. Brasília: Organização das Nações Unidas; 2015 [citado 2019 fev 9]. Disponível em: https://nacoesunidas.org/pos2015/agenda2030/

2. Silveira, MMP. Influência do tempo de remoção da paciente para a maternidade de alta complexidade e desfecho desfavorável [dissertação]. Salvador: Escola Bahiana de Medicina e Saúde Pública; 2015. Disponível em: http://www7.bahiana.edu.br/ jspui/bitstream/bahiana/234/1/Dissertação.\%20 Silveira.\%20Márcia\%20Maria\%20Pedreira\%20 da.\%202015.001.BAHIANA.pdf

3. Thaddeus S, Maine D. Too far to walk: maternal mortality in context. Soc Sci Med [Internet]. $1994 \mathrm{Apr}$ [cited 2018 Dec 8];38(8):1091-110. Available from: http://www.ncbi.nlm.nih.gov/pubmed/804205.

4. Gabrysch S, Campbell OMR. Still too far to walk: literature review of the determinants of delivery service use. BMC Pregnancy Childbirth [Internet]. 2009 Jan [cited 2019 Jan 26];9:34. Available from: http://www.pubmedcentral.nih.gov/articlerender.fcgi? $\operatorname{artid}=2744662 \&$ tool $=$ pmcentrez\&rendertype $=$ abstra ct. doi: 10.1186/1471-2393-9-34 saúde. Em 2013, ano de início do Projeto Meta 35, o terceiro e último óbito materno registrado em Porto Seguro ocorreu no mês de junho. No ano de 2014, o município não registrou nenhum óbito materno, quebrando a sequência de 3 a 4 óbitos/ano. ${ }^{21}$

Sabe-se que o enfrentamento e eliminação da mortalidade materna é tarefa premente e requer esforços contundentes. Também é evidente a necessidade da promoção do bem-estar das mulheres e de melhores índices de sobrevivência e qualidade de vida para seus filhos. Pequenas ações, realizadas no dia a dia de cada profissional de saúde, podem proporcionar resultados concretos na direção de mudanças desse cenário inseguro, porém evitável.

\section{Contribuição das autoras}

Todas as autoras participaram da concepção, delineamento do estudo, análise e interpretação dos dados e redação do manuscrito, com responsabilidade em todos os aspectos do trabalho. Todas aprovaram a versão final do manuscrito.

5. Mccarthy J, Maine D. A framework for analyzing the determinants of maternal mortality. Stud Fam Plann [Internet].1992 Jan-Feb [cited 2019 Jul 8];23(1):23-33. Available from: https://www.jstor.org/ stable/1966825. doi: 10.2307/1966825

6. Calderón IMP, Cecatti JG, Vega CEP. Intervenções benéficas no pré-natal para prevenção da mortalidade materna. Rev Bras Ginecol Obs [Internet]. 2006 maio [citado 2018 nov 9];28(5):310-5. Disponível em: http://www.scielo.br/pdf/rbgo/v28n5/a08v28n5.pdf. doi: 10.1590/S0100-72032006000500008

7. Domingues RMSM, Hartz ZMA, Dias MAB, Leal MC. Avaliação da adequação da assistência pré-natal na rede SUS do Município do Rio de Janeiro, Brasil. Cad Saúde Pública [Internet]. 2012 mar [citado 2018 dez 6];28(3):425-37. Disponível em: http://www. scielo.br/pdf/csp/v28n3/03.pdf. doi: 10.1590/S0102311X2012000300003

8. Ministério da Saúde (BR). Secretaria de Políticas de Saúde, Área Técnica da Saúde da Mulher. Urgências e emergências maternas: guia para diagnóstico e conduta em situações de risco de morte materna [Internet]. 2. ed. Brasília: Ministério da Saúde; 2000 [citado 2019 jul 8]. 119 p. Disponível em: http://bvsms.saude.gov.br/ bvs/publicacoes/0105urgencias.pdf 
9. Ministério da Saúde (BR). Secretaria de Atenção à Saúde. Departamento de Atenção Básica. Atenção ao pré-natal de baixo risco [Internet]. Brasília: Ministério da Saúde; 2012 [citado 2019 jul 8]. 318 p. (Série A. Normas e Manuais Técnicos) (Cadernos de Atenção Básica, ${ }^{\circ}$ 32). Disponível em: http://bvsms. saude.gov.br/bvs/publicacoes/cadernos_atencao_ basica_32_prenatal.pdf

10. Ministério da Saúde (BR). Instituto Sírio-Libanês de Ensino e Pesquisa. Protocolos da atenção básica: saúde das mulheres [Internet]. Brasília: Ministério da Saúde; 2016 [citado 2019 jul 8]. 230 p. Disponível em: http://189.28.128.100/dab/docs/portaldab/ publicacoes/protocolo_saude_mulher.pdf

11. Morse ML, Fonseca SC, Barbosa MD, Calil MB, Eyer FPC. Mortalidade materna no Brasil: o que mostra a produção científica nos últimos 30 anos? Cad Saúde Pública [Internet]. 2011 abr [citado 2018 nov 12];27(4):623-38. Disponível em: http://www. scielo.br/pdf/csp/v27n4/02.pdf. doi: 10.1590/S0102311X2011000400002

12. Ministério da Saúde (BR). Secretaria de Vigilância em Saúde. Departamento de Análise de Situação em Saúde. Guia de vigilância epidemiológica do óbito materno [Internet]. Brasília: Ministério da Saúde; 2009 [citado 2019 jul 8]. 84 p. (Série A. Normas e Manuais Técnicos). Disponível em: http://bvsms. saude.gov.br/bvs/publicacoes/guia_vigilancia_ epidem_obito_materno.pdf

13. Brasil. Ministério da Saúde. Resolução MS/CNS n ${ }^{0}$ 510, de 7 de abril de 2016. Dispõe sobre as normas aplicáveis a pesquisas em Ciências Humanas e Sociais cujos procedimentos metodológicos envolvam a utilização de dados diretamente obtidos com os participantes ou de informações identificáveis ou que possam acarretar riscos maiores do que os existentes na vida cotidiana [Internet]. Diário Oficial da União, Brasília (DF), 2016 maio 24 [citado 2019 jul 8];Seção 1:44-6. Disponível em: http://bvsms.saude.gov.br/bvs/saudelegis/cns/2016/ res0510_07_04_2016.html

14. Faria DR, Sousa RC, Costa TJNM, Leite ICG. Mortalidade materna em cidade-polo de assistência na região Sudeste: tendência temporal e determinantes sociais. Rev Med Minas Gerais [Internet]. 2012 [citado 2018 nov 12];22(1):1128. Disponível em: http://www.rmmg.org/artigo/ detalhes/121.
15. Teixeira NZF, Pereira WR, Barbosa DA, Vianna LAC. Mortalidade materna e sua interface com a raça em Mato Grosso. Rev Bras Saúde Mater Infant [Internet]. 2012 [citado 2019 fev 12];12(1):27-35. Disponível em: http://www.scielo.br/pdf/rbsmi/v12n1/03.pdf. doi: 10.1590/\$1519-38292012000100003

16. Leite RMB, Araújo TVB, Albuquerque RM, Andrade ARS, Duarte Neto PJ. Fatores de risco para mortalidade materna em área urbana do Nordeste do Brasil. Cad Saúde Pública [Internet]. 2011 out [citado 2019 jan 31];27(10):1977-85. Disponível em: http://www.scielo.br/pdf/csp/v27n10/11.pdf. doi: 10.1590/S0102-311X2011001000011

17. Ministério da Saúde (BR). Secretaria de Atenção à Saúde. Coordenação Geral da Saúde das Mulheres. Departamento de Atenção Básica. Caderneta da gestante [Internet]. Brasília: Ministério da Saúde; 2018 [citado 2019 jul 8]. Disponível em: http:// portalarquivos2.saude.gov.br/images/pdf/2018/ agosto/31/Caderneta-da-Gestante-2018.pdf

18. Silva BGC, Lima NP, Silva SG, Antúnez SF, Seerig LM, Restrepo-Méndez MC, et al. Mortalidade materna no Brasil no período de 2001 a 2012: tendência temporal e diferenças regionais. Rev Bras Epidemiol [Internet]. 2016 jul-set [citado 2019 jan 27];19(3):484-93. Disponível em: http://www.scielo.br/pdf/rbepid/ v19n3/1980-5497-rbepid-19-03-00484.pdf. doi: 10.1590/1980-5497201600030002

19. Martins ACS, Silva LS. Epidemiological profile of maternal mortality. Rev Bras Enferm [Internet]. 2018 [cited 2019 Mar 15];71(Suppl 1):677-83. Available from: http://www.scielo.br/pdf/reben/v71s1/pt_00347167-reben-71-s1-0677.pdf. doi: 10.1590/00347167-2017-0624

20. Moura BLA, Alencar GP, Silva ZP, Almeida MF. Internações por complicações obstétricas na gestação e desfechos maternos e perinatais, em uma coorte de gestantes no Sistema Único de Saúde no município de São Paulo, Brasil. Cad Saúde Pública [Internet]. 2018 [citado 2019 mar 19];34(1):e00188016. Disponível em: http:// www.scielo.br/pdf/csp/v34n1/1678-4464-csp-3401-e00188016.pdf. doi: 10.1590/0102-311x00188016.

21. Secretaria de Saúde do Estado da Bahia. Informações em saúde [Internet]. Salvador: Secretaria de Saúde do Estado da Bahia; 201- [citado 2019 jul 8]. Disponível em: http://www.saude.ba.gov.br/suvisa/diretoria-deinformacao-em-saude-dis 


\begin{abstract}
Objective: to portray the creation of a gestational risk classification based on the profile of maternal deaths in a municipality where the rate of these deaths is high, and its use in prenatal care. Methods: the profile was prepared using records of maternal deaths that occurred between 2008 and 2013, considering age, schooling, race/skin color, place of residence, pre-existing disease, reproductive history. Results: maternal death was most frequent in women of brown/ black skin color, aged 30-39, with low schooling, living in socially vulnerable districts and with heart disease as the main pre-existing disease; gestational risk was classified based on this profile, whereby points (1-3) were assigned to each lowest/highest frequency and care priorities $(P)$ were defined $-P I=$ regular risk (4-9pt: routine consultation/examination), PII=high risk (1016pt: reduce waiting time for consultation/examination by $50 \%)$, and PIII=very high risk ( $\geq 17$ pt: access to consultation/examination within 7 days). Conclusion: the new classification improved healthcare professionals' awareness of determinants surrounding maternal death and the need to prioritize access to prenatal care according to risk.
\end{abstract}

Keywords: Maternal Mortality; Risk Factors; Prenatal Care; Health Services Accessibility; Cross-Sectional Studies.

\section{Resumen}

Objetivo: retratar la creación de clasificación de riesgo gestacional basada en el perfil de óbitos maternos en un municipio con alta incidencia y su utilización en el prenatal. Métodos: el perfil fue elaborado analizando fichas de óbitos maternos ocurridos entre 2008-2013, considerando edad, escolaridad, raza/color de piel, domicilio, enfermedad preexistente, historia reproductiva. Resultados: el óbito materno fue mas frecuente en pardas/negras, entre 30-39 años, con baja escolaridad, residentes en distritos socialmente vulnerables y con cardiopatía preexistente; el riesgo gestacional se clasificó a partir del perfil, designando puntos (1-3) para cada menor/mayor frecuencia $e$ definiendo prioridades $(P)$ asistenciales $-P I=$ riesgo habitual (4-9pt: rutina para consultas/exámenes), PII=alto riesgo (10-16pt: tiempo de espera reducido $50 \%$ para consultas/exámenes) y PIII= muy alto riesgo ( $\geq 17 p t$ : acceso a consultas/exámenes en máximo 7 días). Conclusión: la nueva clasificación sensibilizó a los profesionales sobre los determinantes relacionados con esas muertes y la necesidad de dar prioridad al acceso a la atención prenatal considerando el riesgo.

Palabras clave: Mortalidad Materna; Factores de Riesgo; Atención Prenatal; Accesibilidad a los Servicios de Salud; Estudios Transversales.

Recebido em 19/02/2019

Aprovado em 28/06/2019 